\title{
Re: Testosterone Treatment Is a Potent Tumor Promoter for the Rat Prostate:
}

\section{Bosland MC}

\author{
Jason M. Scovell, Peter Butler, Ranjith Ramasamy, and Dolores J. Lamb \\ Department of Urology and Center for Reproductive Medicine, Baylor College of Medicine, \\ Houston, TX, USA
}

\section{Experts' summary}

This animal study compared the effect of testosterone supplementation on prostatic and allsite carcinogenesis in the male rat after androgen deprivation treatment $(n=28-30$ per treatment group). Using various treatment approach models, rats aged 10-12 wk were pretreated with antiandrogen therapy for $3 \mathrm{wk}$, subsequently had testosterone replaced with injectable testosterone propionate for $3 \mathrm{~d}$, and finally were treated with the toxic compound $N$-nitroso- $N$-methylurea (NMU) to induce carcinogenesis. After this pretreatment regimen, various numbers of slowly eluting testosterone implants were placed in the rats, including a placebo. Previous studies have shown prostatic carcinogenesis using a combination of NMU and implantable testosterone; this study aimed to provide dose-response data.

An increase in serum testosterone was only seen for two or more implants $(p<0.001)$, and this response was durable up to $20 \mathrm{wk}(p<0.05)$. The untreated control group had $0 \%$ incidence of prostatic/accessory ( $\mathrm{p} / \mathrm{ag}$ ) gland malignancy, and rats that underwent the pretreatment regimen all had some incidence of $\mathrm{p} / \mathrm{ag}$ tumors. Pretreated rats without testosterone had the lowest incidence of p/ag malignancy (4\%) followed by rats with one tube (57\%), two tubes (71-76\%), and four tubes (76\%). Rats that had two or four implantable tubes had greater rates of $\mathrm{p} / \mathrm{ag}$ adenocarcinoma compared to rats that did not receive implantable testosterone $(p<0.0001)$. In rats without pretreatment therapy, there was a greater incidence of $\mathrm{p} / \mathrm{ag}$ carcinoma in animals with two implantable tubes but not four implantable tubes when compared to controls (four tubes, $13 \%, p=0.06$; two tubes, $25 \%, p$ $<0.05$; 0 tubes, $0 \%$ ).

\section{Experts' comments}

Prostatic hyperplasia and eventual adenocarcinogenesis are dependent on testosterone. This animal model demonstrates the differentiating effect of testosterone on prostatic epithelial tissue. These concerns are not new; indeed, they were the paradigm until relatively recently. Huggins et al [1] raised the initial concerns that exogenous testosterone use could result in

"Corresponding author. Department of Urology and Center for Reproductive Medicine, Baylor College of Medicine, One Baylor Plaza, N 730, Houston, TX 77030, USA. dlamb@ bcm.edu (D.J. Lamb).

Conflicts of interest: The authors have nothing to disclose. 
the generation of new lesions and stimulate existing adenocarcinoma. However, the concern that testosterone supplementation therapy will result in increased levels or severity of prostatic carcinoma has been tempered in recent years [2]. The literature suggests that physiological levels of serum testosterone have already saturated prostatic androgen receptors, and that at supraphysiological levels the stimulatory effect of testosterone on the prostate is negligible [3].

Bosland sought to explore this relationship between testosterone and prostatic adenocarcinoma. This research supports the understanding that testosterone is necessary for the development of prostatic adenocarcinoma, which has been demonstrated by negligible rates of prostate cancer in the eunuch male population [4]. Specifically, this study sought to demonstrate increasing prevalence of prostate cancer in a dose-dependent response to testosterone implants. To stimulate the growth of prostate cancer by testosterone, the strong alkylating agent NMU was used. NMU led to the genesis of other benign and malignant tumors in tissues not associated with androgen action (pituitary adenomas, lymphomas, Zymbal gland carcinomas, skin tumors). Furthermore, carcinogenesis originated in some cases in seminal vesicles, which is distinct in origin from prostate cancer. The clinically relevant information from this study is that the prevalence of prostatic adenocarcinoma did not increase after serum testosterone reached physiological or supraphysiological levels. This is corroborated by the aforementioned research suggesting that testosterone at or above physiological levels has a negligible effect on the prostate.

Bosland also found that exogenous testosterone treatment alone increased the risk of malignant tumors, but this finding has not been confirmed by other studies. Bosland's work builds on a study by McCormick et al [5], who found that testosterone and NMU together increase tumor incidence. However, this study differs from the previous one in that McCormick et al found that testosterone alone (at eugonadal levels) was responsible for a tumor incidence of $<10 \%$ versus Bosland's finding of $25 \%$. It is tempting to speculate that exogenous testosterone administration (even at low doses) was involved in the development of prostate cancer.

Researchers and practitioners in the field should interpret these findings with caution. It is clear that testosterone is critical for the development and progression of prostate adenocarcinoma. However, these results provide further evidence that at or above physiological levels, there is no increase in the risk of carcinogenesis. Further research is also required to determine whether exogenous testosterone alone leads to significantly higher incidence of prostate tumors. Until prospective randomized trials that may be able to answer these questions are completed [6], prostate cancer should remain a risk factor for testosterone supplementation. Our understanding of the literature and experience suggests that there is no clinically appreciable risk with regard to prostatic adenocarcinoma in men treated with testosterone supplementation therapy.

\section{References}

1. Huggins C, Stevens RE Jr, Hodges CV. Studies on prostatic cancer II. The effects of castration on advanced carcinoma of the prostate gland. Arch Surg. 1941; 43:209-23. 
2. Rhoden EL, Morgentaler A. Risks of testosterone-replacement therapy and recommendations for monitoring. N Engl J Med. 2004; 350:482-92. [PubMed: 14749457]

3. Morgentaler A. Goodbye androgen hypothesis, hello saturation model. Eur Urol. 2012; 62:765-7. [PubMed: 22728116]

4. Wynder EL, Laakso K, Sotarauta M, Rose DP. Metabolic epidemiology of prostatic cancer. Prostate. 1984; 5:47-53. [PubMed: 6364075]

5. McCormick DL, Rao KV, Dooley L, et al. Influence of N-methyl-N-nitrosourea, testosterone, and $\mathrm{N}$-(4-hydroxyphenyl)-all-transretinamide on prostate cancer induction in Wistar-Unilever rats. Cancer Res. 1998; 58:3282-8. [PubMed: 9699656]

6. Snyder PJ, Ellenberg SS, Cunningham GR, et al. The testosterone trials: seven coordinated trials of testosterone treatment in elderly men. Clin Trials. 2014; 11:362-75. [PubMed: 24686158] 